\title{
Valeur alimentaire du foin de luzerne pour la chèvre
}

\author{
Sylvie GIGER, D. SAUVANT, J. HERVIEU, Michelle DORLEANS \\ INRA, Station de Nutrition et Alimentation, \\ I.N.A. Paris-Grignon \\ 16, rue Claude-Bernard, F 75231 Paris Cedex 05
}

\begin{abstract}
Résumé
Le foin de luzerne est un fourrage de choix dans l'alimentation des chèvres laitières. Sa valeur nutritive, qui est bien connue chez les ovins, l'est beaucoup moins chez les caprins. Les résultats de cette étude sur caprins montrent, qu'à composition d'ingéré équivalente, les chèvres taries ingèrent significativement moins $\left(-10,4 \mathrm{~g} \mathrm{MS} / \mathrm{kg} \mathrm{P^{0.75 }}\right.$ ) que les moutons (MCS, ANDRIEU et al., 1981). Cependant, les animaux en lactation ont une capacité d'ingestion plus élevée que celle des moutons castrés standards (MCS) d'environ $30 \%$. Les chèvres digèrent en moyenne mieux $(+1,5$ point de $\mathrm{dMO}$ ) le foin que les moutons de référence. Aucune différence de la dMO n'a pu être mise en évidence, avec des foins différents, mais également équivalents en terme de composition de l'ingéré, entre chèvres taries et en production.

Le taux moyen de refus de $15 \%$, par rapport à l'offert, se traduit par une amélioration moyenne de la digestibilité de la matière organique de l'ingéré par rapport au distribué de 0.6 point. La quantité de matière sèche ingérée augmente, pour un foin donné, avec la production laitière. Mais, les différences d'ingestion observées entre les trois foins considérés ne sont liées ni à leur digestibilité, ni à leur composition ou au besoin énergétique des animaux, ce qui soulève le problème de l'application des unités d'encombrement.
\end{abstract}

Mots clés: Chèvre laitière, foin de luzerne, valeur nutritive.

\section{Introduction}

Le foin de luzerne jouit d'une bonne réputation pour alimenter les chèvres, en raison notamment de son ingestibilité élevée et de l'apport azoté qu'il constitue. Cependant, aucune étude systématique de la valeur alimentaire de ce foin pour la chèvre n'a, à notre connaisance, été effectuée bien que des questions spécifiques et importantes demeurent posées à ce propos :

- Peut-on appliquer aux chèvres les caractéristiques de valeur alimentaire du foin de luzerne observées chez les moutons castrés standard (MCS) qui ont servi à l'élaboration des tables de l'INRA de 1978 (Demarquilly, Andrieu \& Sauvant, 1978) et de 1981 (Andrieu, Demarquilly \& Wegat-Litre, 1981)?

- Quelle est l'influence du tri sur la valeur nutritive de la fraction de fourrage effectivement ingérée?

- Quelle est l'influence des variations de la capacité d'ingestion des animaux, en fonction de leur stade physiologique, sur la digestibilité du foin de luzerne? 
- Quelle est la production laitière maximale permise par le foin distribué comme seul aliment?

Pour apporter des éléments de réponse, le travail présenté regroupe les résultats de mesures de digestibilité et d'ingestibilité effectuées sur sept foins de luzerne différents distribués en aliment unique à des chèvres taries ou en lactation.

\section{Conditions expérimentales}

Les mesures, effectuées avec du foin seul, avaient pour objet principal de connaître leur digestibilité. Sept livraisons de foin de luzerne ont ainsi été étudiées avec des chèvres en lactation ou taries (gravides ou vides) selon les modalités décrites au tableau 1. La digestibilité de chaque foin a été mesurée sur 3 à 6 animaux, soit de 3 à 12 fois, si l'on tient compte des répétitions des mesures. Au total, 51 observations individuelles ont été effectuées sur les 7 foins. Chacune correspond à des mesures d'ingestion et de digestibilités faites pendant cinq jours consécutifs. Sur les différents échantillons de foin et de fèces, les déterminations suivantes ont été effectuées : matière sèche, cendres, cellulose brute, matières azotées totales, paroi végétale (NDF) de VAN Soest \& Wine (1967), lignocellulose (ADF) et lignine (ADL) de VAN Soest (1963). Lorsque le refus excède 5 p. 100 de la quantité distribuée, il est l'objet d'une analyse chimique spécifique afin de déterminer, par différence, la composition précise de l'ingéré.

Les 31 animaux qui ont participé à ces expériences sont de race alpine chamoisée ou Saanen. Ils sont placés dans les cages à digestibilité décrites par Giger \& Hervieu (1980) et sont soumis au protocole habituellement utilisé à la Station INRA de Nutrition et Alimentation de l'INA-PG (GIGER et al., 1982). Le poids moyen de ces animaux, âgés en moyenne de 4 ans, est de $59,3 \mathrm{~kg}$. Les 16 chèvres en production sont toutes des adultes, ayant déjà accompli au moins une lactation entière. Elles produisent en moyenne $1,73 \mathrm{~kg}$ de lait corrigé à $35 \mathrm{p}$. 1000 de taux butyreux par jour (PL35) et sont en phase descendante de lactation ( $15^{\mathrm{e}}$ à la $32^{\mathrm{e}}$ semaine après la mise-bas) avec un stade moyen qui se situe à 6 mois de la parturition.

L'interprétation des résultats a notamment été effectuée à l'aide de l'étude des corrélations inter et intra foins entre les caractéristiques mesurées et d'un programme d'analyse de variance et covariance qui permet de tester les effets des régimes (types de foin) et ceux des covariables suivant les modalités décrites par SNEDECor et CocHRAN (1984).

\section{Résultats}

\section{A. Caractéristiques moyennes des foins ingérés}

Par rapport aux tables INRA 1978, (Demarquilly, Andrieu \& Sauvant, 1978), les foins étudiés peuvent être considérés comme étant de bonne valeur nutritive puisqu'ils ont en moyenne des teneurs de 30,7 et 17,1 p. 100 de cellulose brute (CB) et de matières azotées totales (MAT) par rapport à la matière sèche respectivement (tabl. 1). 


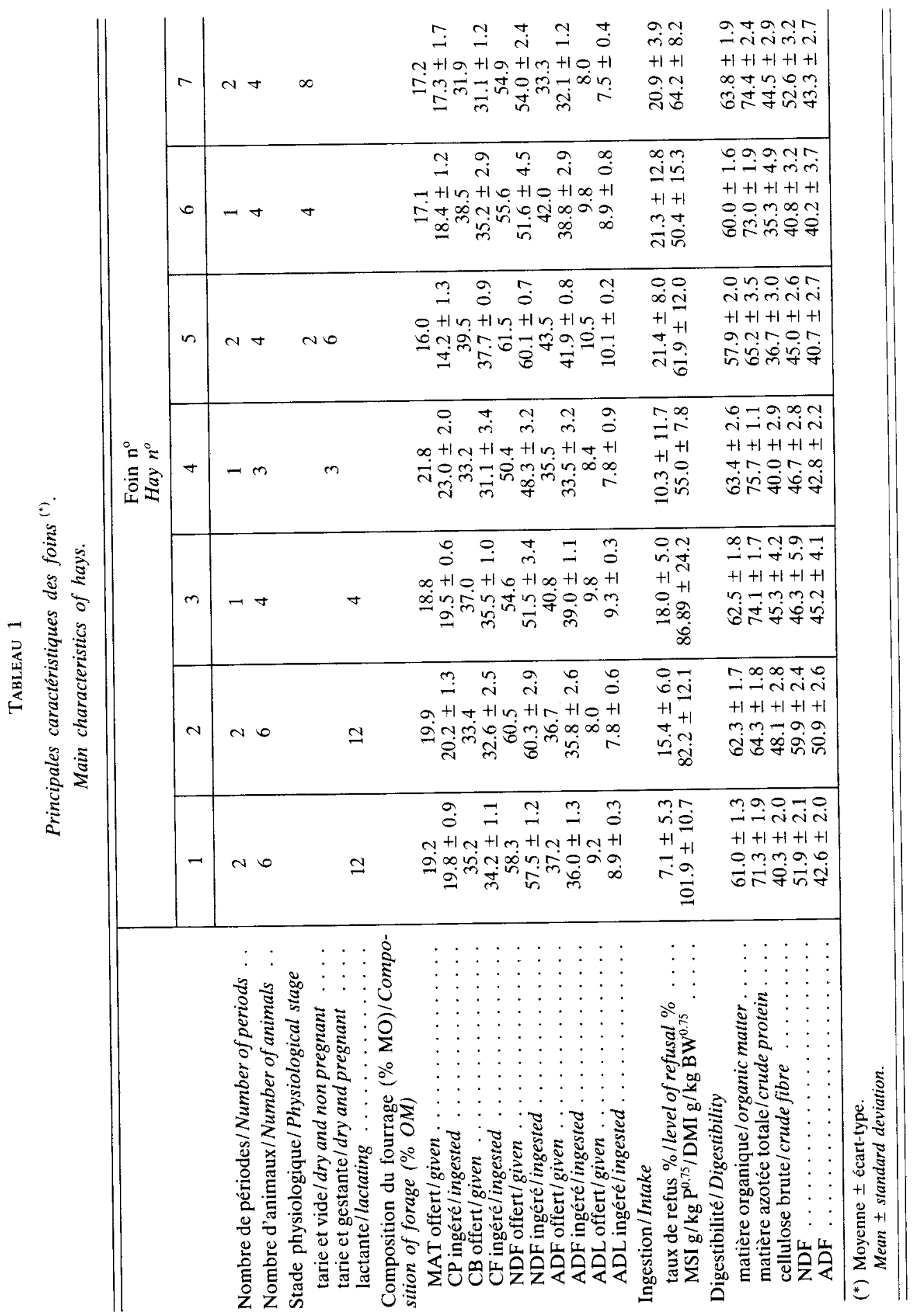


La digestibilité de la matière organique ( $\mathrm{dMO}$ ) des trois foins ingérés par les chèvres en lactation a été en moyenne de 61,8 p. 100. Cette moyenne est de 61,0 p. 100 pour les quatre foins ingérés par les chèvres taries. Ces valeurs comparables sont cohérentes avec les teneurs moyennes équivalentes en cellulose brute $(34,1$ p. 100 et $33,8$ p. $100 / \mathrm{MO})$ et en matières azotées totales $(19,9$ et $17,1 \mathrm{p} .100 / \mathrm{MO})$ des foins ingérés par ces deux types d'animaux. Les valeurs de dMO calculées à partir de l'équation obtenue sur les MCS (Andrieu, Demarquilly \& Wegat-Litre, 1981) sont étroitement liées $(r=0,88)$ aux valeurs mesurées (tabl. 2) ; cependant, la dMO des chèvres est, pour une même composition de foin, en moyenne, significativement supérieure $(\mathrm{P}<0,01)$ de 1,5 point à celle obtenue sur les moutons.

Les quantités moyennes de matière sèche ingérée (MSI) sont bien plus importantes $(+53$ p. 100) pour les animaux en lactation que pour les animaux taris : 91,3 contre $59,8 \mathrm{~g} \mathrm{MSI} / \mathrm{kg} \mathrm{P}^{0.75}$, soit environ 2,0 et $1,3 \mathrm{~kg}$ de MS par animal, respectivement. Les ingestions des chèvres en production sont nettement supérieures, de $22,4 \mathrm{~g} / \mathrm{kg} \mathrm{P} \mathrm{P}^{0.75}$, soit 33 p. 100, à celles prédites par l'équation INRA 1981 obtenue sur les moutons MCS (tabl. 2). Les niveaux d'ingestion des chèvres taries sont, pour les quatre foins, statistiquement inférieurs de $10,4 \mathrm{~g} / \mathrm{kg}$ de $P^{0.75}$ à ceux estimés pour des moutons castrés standard. Les pourcentages de refus des chèvres ont varié, dans ce cas, de 10 à $30 \mathrm{p}$. 100 de la matière sèche offerte.

TABleau 2

Comparaison des valeurs observées sur chèvres avec celles calculées à partir des relations de prédiction obtenues sur des MCS.

Comparison between values observed in goats and those calculated from sheep equations.

\begin{tabular}{|c|c|c|c|c|c|c|}
\hline \multirow[b]{2}{*}{$\begin{array}{l}N^{\prime \prime} \text { du foin } \\
N^{o} \text { of hay }\end{array}$} & \multicolumn{2}{|c|}{$\begin{array}{c}\text { Composition chimique } \\
\text { p. } 100 \mathrm{Mo} \\
\text { Chemical composition } \\
\text { p. } 100 \mathrm{OM}\end{array}$} & \multicolumn{2}{|c|}{ 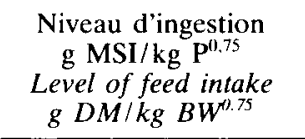 } & \multicolumn{2}{|c|}{$\begin{array}{l}\text { Digestibilité de } \\
\text { la MO p. } 100 \\
\text { Digestibility of } \\
\text { the OM p. } 100\end{array}$} \\
\hline & $\begin{array}{l}\mathrm{CB} \\
C F\end{array}$ & $\begin{array}{c}\text { MAT } \\
C P\end{array}$ & $\begin{array}{c}\text { Mesuré } \\
\text { Measured }\end{array}$ & $\begin{array}{l}\text { Prédit } \\
\text { Predicted }\end{array}$ & $\begin{array}{l}\text { Mesurée } \\
\text { Measured }\end{array}$ & $\begin{array}{l}\text { Prédite } \\
\text { Predicted }\end{array}$ \\
\hline 1 & 34.2 & 19.8 & $101.9^{(1)}$ & 67.5 & 61.0 & 60.1 \\
\hline 2 & 32.6 & 20.2 & $82.2^{(1)}$ & 70.1 & 62.2 & 60.9 \\
\hline 3 & 35.5 & 19.5 & $86.8^{(1)}$ & 66.0 & 62.5 & 59.4 \\
\hline 4 & 31.1 & 23.0 & 55.0 & 71.4 & 63.4 & 62.2 \\
\hline 5 & 37.7 & 14.2 & 61.9 & 64.2 & 57.9 & 57.3 \\
\hline 5 & 35.2 & 18.4 & 50.4 & 66.2 & 60.0 & 59.3 \\
\hline 7 & 31.1 & 17.3 & 64.2 & 71.5 & 63.8 & 61.0 \\
\hline
\end{tabular}

(1) Mesures effectuées sur des animaux en lactation./Measurements done in lactating goats.

(2) A partir de l'équation (ANDRIEU et al., 1981)./From the equation (ANDRIEU et al., 198I).

$\mathrm{MSI} / \mathrm{P}^{0.75}=105,94-1,230 \mathrm{CB}(\mathrm{ETR}=7,7, \mathrm{r}=0,552) / \mathrm{DMI} / \mathrm{BW}^{0.75}=105.94-1.230 \mathrm{CF}(\mathrm{RSD}=7.7, \mathrm{r}=0.552)$ $\mathrm{CB}:$ p. 100 de la matière sc̀che/CF : p. 100 of dry matter.

(3) Pour le MCS, à partir de l'équation (Andrieu et al., 1981)./For sheep, from the (ANDRIEu et al., 1981) equation. $\mathrm{dMO}=71,5+0,216 \mathrm{MAT}^{\circ}-0,459 \mathrm{CB}^{\circ}(\mathrm{ETR}=3,0, \mathrm{r}=0,576)$

$\mathrm{OMD}=71.5+0.216 \mathrm{CP}^{\mathrm{0}}-0.459 \mathrm{CF}^{\circ}(\mathrm{RSD}=3.0, \mathrm{r}=0.576)$

$\mathrm{MAT}^{\circ}, \mathrm{CB}^{\circ}$ : p. 100 de la matic̀re organique/CP, $\mathrm{CF}^{\circ}$ : p. 100 of organic matter. 


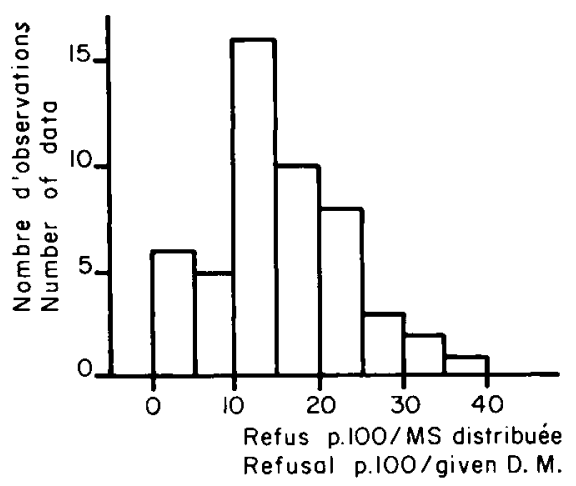

FIG. 1

Histogramme des pourcentages de refus mesurés individuellement.

Histogramm of measured individually percentages of refusals.

B. Influence du tri des animaux sur la digestibilité du foin de luzerne

La proportion moyenne de refus est de 15,7 p. 100, avec des différences individuelles importantes (fig. 1), puisque le coefficient de variation entre les individus, à l'intérieur d'une même expérience, c'est-à-dire pour un protocole de distribution identique, est de 43,6 p. 100. La figure 2 traduit l'effet du tri des animaux qui ingèrent, de ce fait, un foin de qualité supérieure au distribué puisque la teneur en cellulose brute a diminué en moyenne de 1,3 p. 100/MO et celle des matières azotées totales a augmenté de 0,15 p. 100/MO. Les écarts-types intra-foin des teneurs en cellulose brute,

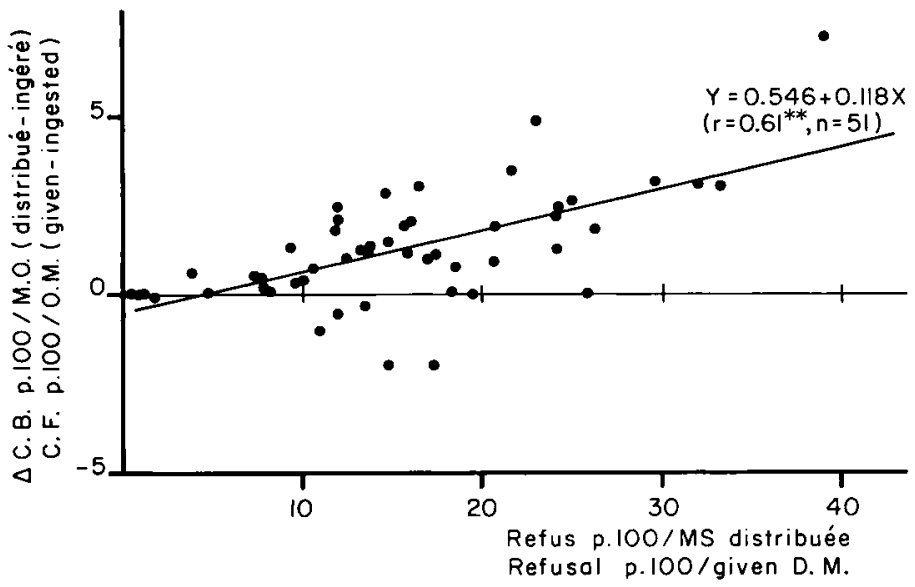

FIG. 2

Relation entre le taux de refus et la différence de la teneur en cellulose brute entre les quantités distribuées et ingêrées.

Relation between the refusal level and the variation in crude fibre content between given and ingested hay. 
NDF, ADF, ADL et MAT de l'ingéré moyen sont respectivement de 1,85, 2,36, 1,92, 0,47 et 1,28 p. $100 / \mathrm{MO}$, ce qui montre l'impact du tri, pour un foin donné, effectué par les animaux sur la qualité du foin ingéré. Les variations de ces teneurs en constituants pariétaux sont positivement et significativement corrélées entre elles. Elles sont négativement corrélées au pourcentage de refus (fig. 2) et à la teneur en MAT (tabl. 3), alors qu'il n'y a pas de liaison significative entre le taux de MAT et le pourcentage de refus (tabl. 3 ).

Les variations intra-foin de la composition de l'ingéré ne sont pas corrélées aux variations individuelles du niveau d'ingestion dont l'écart-type intra-foin est de $12,5 \mathrm{~g}$ $\mathrm{MS} / \mathrm{kg}$ de $\mathrm{P}^{0,75}$, ce qui correspond à un coefficient de variation de $16 \mathrm{p}$. 100. Par contre, lorsque la chèvre réduit, par son tri, la teneur en constituants pariétaux du foin ingéré $(\triangle C B=C B$ distribué $-C B$ ingéré) (corrélation intra $\triangle C B-$ p. 100 refus : 0,75 ) et accroît le taux azoté $(\triangle \mathrm{MAT}=$ MAT distribué - MAT ingéré) (corrélation intra $\triangle \mathrm{MAT}-$ p. 100 refus : $-0,29)$, il y a une amélioration significative de la $\mathrm{dMO}$ du foin. La relation :

$\mathrm{dMO}=76,9-0,453 \mathrm{CB}$ p. 100/MO $+\mathrm{Fi}(\mathrm{r}=0,82, \mathrm{i}=1$ à $7, \mathrm{ETR}=1,60)$ qui relie la dMO au taux de $\mathrm{CB}$ en prenant en compte un effet lié au foin (Fi, avec i variant de 1 à 7) indique que, par son tri, la chèvre améliore de 0,453 point la dMO du foin par point de diminution de la teneur en cellulose brute. Avec les données considérées, la diminution moyenne de 1,30 p. 100/MO correspond donc à une amélioration de 0,60 point de la dMO, soit 0,01 UFL. Dans les situations de tri maximal observées (refus $=40$ p. $100 / \mathrm{MS}$ distribuée), le foin de luzerne ingéré a une digestibilité supérieure de 3,3 points en moyenne, au foin distribué, ce qui correspond à $0,05 \mathrm{UFL} / \mathrm{kg}$ de MS. Les corrélations intra-foin entre la dMO et les teneurs en ADF et ADL de l'ingéré ne sont que très légèrement supérieures à celles obtenues avec la cellulose brute (tabl. 4). La prévision de la dMO n'est que très légèrement améliorée par la prise en compte de la qualité du refus puisque les écarts-types résiduels des régressions liant la dMO aux teneurs en cellulose brute de l'ingéré et du distribué sont respectivement de 1,70 et

\section{TABLEAU 3}

Corrélations intra foin entre les principales caractéristiques mesurées (44 d.d.l.).

Within-hay correlations between the main measured characteristics (44 d.f.).

\begin{tabular}{|c|c|c|c|c|c|c|}
\hline${ }_{C F}^{\mathrm{CB}} \cdots \cdots \cdots$ & 1.00 & & & & & \\
\hline$\underset{N D F}{\mathrm{NDF}} \cdots \ldots \ldots$ & $0.85^{\circ}$ & 1.00 & & & & \\
\hline $\begin{array}{l}\mathrm{ADF} \ldots \ldots \ldots \\
A D F\end{array}$ & $0.99^{\circ 0}$ & $0.88^{\circ 0}$ & 1.00 & & & \\
\hline $\begin{array}{l}\mathrm{ADL} \\
A D L\end{array} \cdots \cdots \cdots$ & $0.94^{\infty}$ & $0.92^{\circ}$ & $0.96^{\circ}$ & 1.00 & & \\
\hline$\underset{C P}{\operatorname{MAT}} \ldots \ldots \ldots$ & $-0.60^{\mathrm{oo}}$ & $-0.29^{\infty}$ & $-0.58^{\infty}$ & $-0.48^{\infty 0}$ & 1.00 & \\
\hline $\begin{array}{l}\text { REFUS } \\
\text { REFUSAL }\end{array}$ & $-0.61^{\infty}$ & $-0.46^{\mathrm{oo}}$ & $-0.60^{\infty}$ & $-0.63^{\circ o}$ & 0.21 & 1.00 \\
\hline $\begin{array}{l}\text { p. } 100 \cdots \cdots \cdots \\
\text { p. } 100\end{array}$ & $\begin{array}{l}\text { CB } \\
C F\end{array}$ & $\begin{array}{l}\text { NDF } \\
N D F\end{array}$ & $\begin{array}{l}\text { ADF } \\
A D F\end{array}$ & $\begin{array}{l}\mathrm{ADL} \\
A D L\end{array}$ & $\begin{array}{c}\text { MAT } \\
C P\end{array}$ & $\begin{array}{l}\text { REFUS } \\
\text { REFUSAL }\end{array}$ \\
\hline
\end{tabular}

Niveau de signification : $1 \%$ क

Level of significance : $5 \%$ कo 
1,81 point. Cette comparaison pose la question de l'opportunité de l'analyse des refus, dans le cas de la prévision de la dMO du foin de luzerne, du moins quand il est de bonne qualité.

Il n'y a pas de relation intra-foin entre la dMO et le niveau alimentaire exprimé en $\mathrm{kg}$ de MS $(\mathrm{r}=-0,11)$ ou MOD $(r=0,05)$ ingérés par kilogramme de poids métabolique ; ce résultat est cohérent avec l'absence de relation intra-foin citée plus haut entre le niveau d'ingestion et la composition de l'ingéré. Les variations intra de la digestibilité des fractions $\mathrm{CB}$, NDF et ADF sont fortement et positivement corrélées entre elles (elles expliquent environ la moitié des variations de la $\mathrm{dMO}$ ) et, par contre, ne sont pas liées à la digestibilité des fractions NDS (MO-NDF) ou NDS-MAT (MO-NDFMAT) (tabl. 5). Comme dans le cas des matières sèche et organique, ces différentes digestibilités ne sont pas affectées par le niveau alimentaire, qu'il soit exprimé en grammes de MS ou de MOD par kilogramme de poids métabolique.

\section{TABleau 4}

Corrélations intra-foin entre la dMO et les principales caractéristiques analytiques et le taux de refus (44 d.d.l.).

Within-hay correlations between OMd and the main analytical parameters and the level of refusal.

\begin{tabular}{|c|c|c|c|c|c|c|}
\hline $\begin{array}{l}\text { p. } 100 \\
\text { p. } 100\end{array}$ & $\begin{array}{l}\mathrm{CB} \\
\mathrm{CF}\end{array}$ & $\begin{array}{l}\text { NDF } \\
N D F\end{array}$ & $\begin{array}{l}\mathrm{ADF} \\
A D F\end{array}$ & $\begin{array}{l}\mathrm{ADL} \\
A D L\end{array}$ & $\underset{C P}{\mathrm{MAT}}$ & $\begin{array}{c}\text { REFUS } \\
\text { REFUSAL }\end{array}$ \\
\hline $\begin{array}{l}\mathrm{dMO} \\
O M d\end{array}$ & $-0.48^{\circ}$ & $-0.35^{\circ o}$ & $-0.49^{\circ 0}$ & $-0.52^{\infty 0}$ & 0.24 & $0.43^{\circ \circ}$ \\
\hline
\end{tabular}

Niveau de signification : $1 \%{ }^{\text {co }}$

Level of significance : $5 \% "$

TABleAU 5

Corrélations intra-foin entre les digestibilités des principaux constituants (44 d.d.l.). Within-hay correlations between the digestibilities of the main analytical parameters (44 d.f.).

\begin{tabular}{|c|c|c|c|c|c|c|c|}
\hline $\begin{array}{l}\mathrm{dCB} \ldots \ldots \\
C F d\end{array} \ldots$ & 1.00 & & & & & & \\
\hline $\begin{array}{l}\mathrm{dNDF} \ldots \ldots \\
N D F d\end{array}$ & $0.81^{\circ 0}$ & 1.00 & & & & & \\
\hline $\begin{array}{l}\mathrm{dADF} \\
A D F d\end{array}$ & $0.95^{\circ 0}$ & $0.90^{\circ 0}$ & 1.00 & & & & \\
\hline$\underset{C P d}{\mathrm{dMAT}} \ldots \ldots$ & 0.27 & $0.48^{\infty}$ & $0.40^{\circ}$ & 1.00 & & & \\
\hline $\begin{array}{l}\text { dNDS } \ldots \ldots \\
\text { NDSd }\end{array}$ & -0.04 & -0.13 & -0.06 & 0.14 & 1.00 & & \\
\hline $\begin{array}{l}\text { d (NDS-MAT) } \\
(N D S-C P) d\end{array}$ & -0.17 & $-0.33^{\circ}$ & -0.24 & -0.17 & $0.93^{\circ}$ & 1.00 & \\
\hline dMO $\ldots \ldots$ & $0.64^{\infty}$ & $0.75^{\infty}$ & $0.71 \%$ & $0.43^{\infty}$ & $0.45^{(1)}$ & 0.23 & 1.00 \\
\hline & $\begin{array}{l}\mathrm{dCB} \\
C F d\end{array}$ & $\begin{array}{l}\mathrm{dNDF} \\
\text { NDFd }\end{array}$ & $\begin{array}{l}\mathrm{dADF} \\
A D F d\end{array}$ & $\begin{array}{c}\mathrm{dMAT} \\
\mathrm{CPd}\end{array}$ & $\begin{array}{l}\text { dNDS } \\
N D S d\end{array}$ & \multicolumn{2}{|c|}{$\begin{array}{c}\text { d (NDS-MAT) } \\
(N D S-C P) d\end{array}$} \\
\hline
\end{tabular}

Niveau de signification : $1 \%$

Level of significance : $5 \%$ " 
Pour préciser les relations intra-foin décrites, une analyse en composantes principales a été appliquée au tableau des corrélations associant onze des caractéristiques mesurées. Le tableau 6 rapporte les valeurs des corrélations entre ces caractéristiques et les quatre premières composantes principales qui expliquent 90,6 p. 100 de la variation. La première composante traduit l'effet évoqué du tri sur la composition et la digestibilité des différentes fractions du foin ingéré ; le niveau alimentaire n'est pas représenté par cette composante, mais il explique par contre l'essentiel de la troisième composante principale. Les composantes principales 2 et 4 traduisent des aspects qui n'apparaissent pas de façon évidente à l'examen du tableau des corrélations intra. La seconde composante traduit des variations non expliquées par la première de la digestibilité des constituants pariétaux. Plus précisément, elle indique que les variations intra de la digestibilité des constituants pariétaux ne sont pas systématiquement opposées à celles des teneurs. La quatrième composante module également des caractéristiques de la première au niveau de la relation associant le taux azoté à la digestibilité de la fraction soluble dans le détergent neutre de VAN SoEst (dNDS) : lorsque le tri est important le taux azoté et la digestibilité du NDS augmentent, mais la quatrième composante indique que ces deux caractéristiques sont opposées lorsque la chèvre n'opère pas de tri.

\section{TABleau 6}

Analyse en composantes principales des relations intra-foin des principales caractéristiques considérées.

Principal component analysis of within-hay relations of the main characteristics.

\begin{tabular}{|c|c|c|c|c|}
\hline $\begin{array}{l}\text { Composantes principales } \\
\text { Principal components }\end{array}$ & 1 & 2 & 3 & 4 \\
\hline $\begin{array}{l}\text { Part de variation expliquée p. } 100 / \text { Explained } \\
\text { variation part } p .100 \ldots \ldots \ldots \ldots \ldots\end{array}$ & 34.8 & 25.3 & 17.6 & 12.9 \\
\hline \multicolumn{5}{|l|}{ Caractéristiques/Characteristics } \\
\hline $\mathrm{MOD} / \mathrm{P}^{0.75} / \mathrm{DOM} / B W^{0.75}$ & 0.05 & -0.02 & 0.97 & -0.15 \\
\hline MOND $/ \mathrm{P}^{01.75} / \mathrm{NDOM} / B W^{0.75}$ & 0.37 & -0.18 & 0.90 & 0.01 \\
\hline NDFp. $100 / \mathrm{MO} / N D F$ p. $100 / O M$ & 0.71 & 0.66 & -0.06 & 0.07 \\
\hline ADF p. $100 / \mathrm{MO} / A D F p .100 / O M \ldots \ldots$ & 0.87 & 0.42 & 0.01 & -0.19 \\
\hline ADL p. $100 / \mathrm{MO} / A D L$ p. $100 / O M \ldots \ldots$ & 0.87 & 0.47 & 0.01 & -0.04 \\
\hline MAT p. $100 / \mathrm{MO} / C P$ p. $100 / O M$ & -0.57 & 0.06 & 0.25 & 0.74 \\
\hline $\mathrm{dMO} / O M d \ldots \ldots \ldots \ldots$ & -0.83 & 0.40 & 0.04 & -0.35 \\
\hline $\mathrm{dMAT} / C P d$ & -0.43 & 0.49 & 0.29 & 0.15 \\
\hline $\mathrm{dNDF} / N D F d$ & -0.49 & 0.84 & -0.01 & 0.03 \\
\hline $\mathrm{dADF} / A D F d$ & -0.33 & 0.86 & 0.09 & -0.20 \\
\hline $\mathrm{dNDS} / N D S d$ & -0.38 & -0.28 & 0.08 & -0.79 \\
\hline
\end{tabular}

\section{Influence du niveau de production de lait}

Pour l'ensemble des 28 mesures effectuées avec trois foins sur des animaux en lactation, il n'apparaît pas de relation significative entre les niveaux de production de lait (PL35) et de consommation de MS du foin (MSI) ; les relations intra-foin entre ces caractéristiques sont, par contre significatives :

$$
\mathrm{MSI} / \mathrm{P}^{0.75}=34,1+0,707 \mathrm{PL} 35 / \mathrm{P}^{0.75}+\mathrm{Fi}(\mathrm{r}=0,88, \mathrm{i}=(1,3), \mathrm{ETR}=8,0) .
$$


Les relations, illustrées à la figure 3 dans le cas de la MSI, indiquent que la capacité d'ingestion d'une chèvre de $60 \mathrm{~kg}$, au sixième mois de lactation, alimentée uniquement au foin de luzerne s'accroît en moyenne de $0,71 \mathrm{~kg}$ de MSI par kilogramme de lait à 35 p. 1000 de TB supplémentaire. Il convient de remarquer que la hiérarchie, entre les trois foins, des niveaux d'ingestion mesurés pour un même niveau de production de lait n'est pas associée à leurs caractéristiques moyennes de composition, de digestibilité mesurée et d'ingestibilité prédite (tabl. 2). Ainsi, pour une production de $80 \mathrm{~g}$ de lait à $35 \mathrm{p} .1000$ de taux butyreux par $\mathrm{kg}$ de $\mathrm{P}^{(1.75}$, soit $1,7 \mathrm{~kg} /$ animal, les niveaux moyens d'ingestion des foins 1,2 et 3 sont respectivement de 110 , 73 et $88 \mathrm{~g} \mathrm{MS} / \mathrm{kg} \mathrm{P} \mathrm{P}^{0,75}$, soit $2,4,1,6$ et $1,9 \mathrm{~kg} \mathrm{MS} /$ animal. Cette hiérarchie n'est pas cohérente avec les teneurs en cellulose brute ou les digestibilités de la matière organique de ces foins (tabl. 2). En outre, le stade physiologique et le taux de refus ne sont pas associés à cette relation intra-foin liant la production laitière à la quantité de matière sèche ingérée. Par contre, le passé alimentaire des animaux pourrait avoir une influence, puisque pendant les semaines précédant la période de régime foin seul, les animaux ingérant le foin $\mathrm{n}^{\circ} 1$ ont reçu une alimentation plus restreinte que les autres, pendant les deux mois précédents, ce qui pourrait expliquer leur capacité d'ingestion supérieure, en raison d'un besoin plus important de reconstitution des réserves. Cette hypothèse est étayée par le fait que les bilans énergétiques moyens des animaux sont

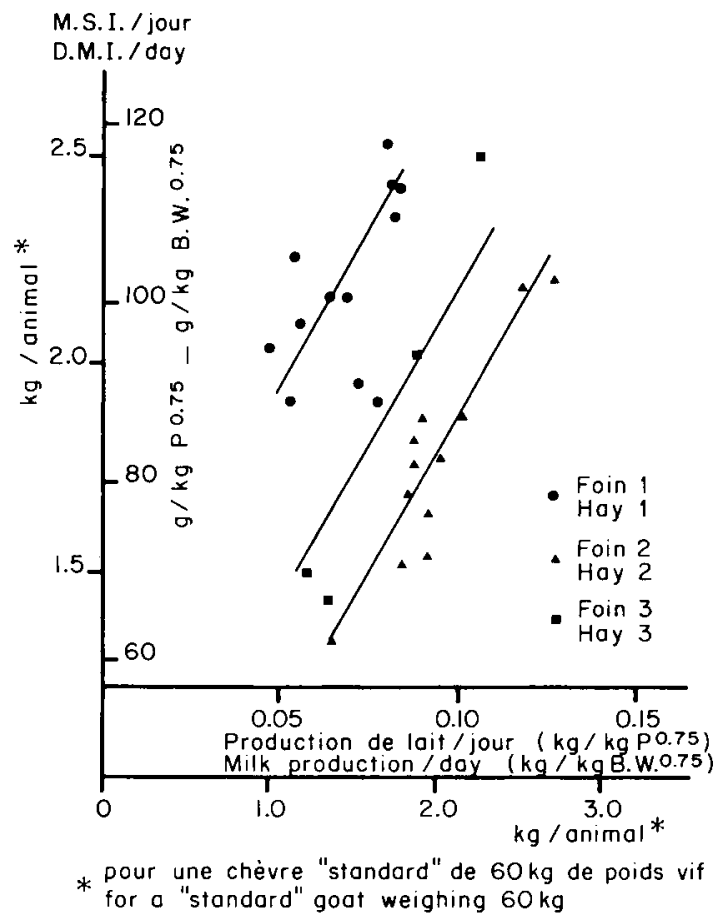

Fig. 3

Relation entre la production laitière à 35 p. 1000 de T.B. et la quantité de matière sèche de foin ingéré.

Relation between corrected milk production (35 p. 1000 milk fat content) and the level of ingested hay dry matter. 
statistiquement différents pour les trois foins : 4,94 $\mathrm{g}$ BMOD $/ \mathrm{kg} \mathrm{P}^{0.75}$ pour le foin 1 , $-14,92$ pour le 2 et $-6,08$ pour le 3 . De plus, les animaux qui produisent le plus ont des bilans statistiquement plus élevés que les autres.

Les variations intra-foin de niveau de production et de capacité d'ingestion ne sont pas corrélées à celles de la digestibilité mesurée de la matière organique ou des constituants pariétaux.

Comme pour la matière sèche ingérée, la matière organique digestible ingérée est corrélée à la production laitière corrigée :

$$
\mathrm{MODI} / \mathrm{P}^{0.75}=20,4+0,378 \mathrm{PL} 35 / \mathrm{P}^{0.75}+\mathrm{Fi}(\mathrm{r}=0,86, \mathrm{i}=(1,3), \mathrm{ETR}=5,0) .
$$

D'après cette équation, la capacité d'ingestion d'une chèvre de $60 \mathrm{~kg}$ est augmentée de $0,378 \mathrm{~kg}$ de MOD par $\mathrm{kg}$ de PL35. Cette dernière valeur est statistiquement supérieure à $0,324 \mathrm{~kg}$ de MOD, qui représente la valeur recommandée du besoin par kilogramme de lait à 35 p. 1000 de taux butyreux (SAuvant, 1981). Elle peut s'expliquer par le fait que les animaux à fort potentiel ont mobilisé davantage en début de lactation, ce qui a entraîné une nécessité pour eux de récupérer plus que les autres (Sauvant et al., 1981). Ce coefficient élevé peut expliquer, en partie, la faible valeur observée pour la constante de l'équation de régression qui représente le besoin d'entretien des animaux.

\section{Discussion et conclusion}

La portée des résultats obtenus est limitée par le nombre et la relative homogénéité des foins expérimentés ; des études devront donc être poursuivies sur ce thème. Les quantités de matière sèche ingérées par les chèvres taries sont statistiquement inférieures à celles estimées sur des moutons castrés standards (MCS ; ANDRIEU et al., 1981), ce qui confirme la comparaison de Jones et al. (1972) qui a également été faite sur du foin de luzerne distribué à des chèvres ou des brevis taries et vides. Cependant, les revues de De Simiane et al. (1981), Brown \& Johnson (1984) et Morand-Fenr et al. (1987) montrent que de telles différences entre ovins et caprins ne sont pas systématiques dans le cas des fourrages secs. En outre, l'état gravide d'une partie des animaux utilisés est susceptible d'expliquer les différences présentement observées ; de plus, le taux de refus toléré, en moyenne de $15 \mathrm{p}$. 100 , est relativement faible pour les chèvres et peut expliquer leur faible niveau d'ingestion (DE Simiane et al., 1981). Ce niveau de refus est cependant proche de celui accepté pour les mesures sur MCS (ANDrieu et al., 1981) qui est de l'ordre de 10 p. 100.

D'après nos résultats, la digestibilité de la matière organique, mesurée sur chèvres, est légèrement, mais systématiquement supérieure, à celle prévue pour une composition d'ingéré équivalente chez des ovins (MCS ; ANDRIEU et al., 1981), ce qui confirme les tendances observées par DE SimIANE et al. (1981).

L'influence du tri des animaux a modifié sensiblement la valeur nutritive du foin de luzerne, malgré le faible taux de refus moyen. Cette influence est variable suivant les individus; elle ne semble pas dépendre de leur capacité d'ingestion, mais du niveau de refus permis et des habitudes de tri des animaux, dernier aspect déjà rapporté par MoRAND-Fehr (1981). L'amélioration obtenue par le tri a pu aller jusqu'à 5 p. 100 de la digestibilité de la matière organique du foin. 
Les chèvres en lactation produisent en moyenne $1,73 \mathrm{~kg} /$ jour et ingèrent en moyenne 50 p. 100 de MS de plus que les chèvres taries. Cette différence de capacité d'ingestion est liée à un écart du niveau des besoins. Il est à noter que la quantité de matière organique digestible ingérée augmente, en moyenne, plus vite que les besoins moyens de lactation habituellement utilisés $(0,378 \mathrm{vs} 0,324 \mathrm{~kg} \mathrm{MOD} / \mathrm{kg}$ de lait à $35 \mathrm{p}$. $1000)$. Cette différence pourrait traduire un besoin de reconstitution plus élevé pour les animaux possédant le potentiel de production le plus important ou le fait que le besoin moyen (SAuvant, 1981) est estimé à partir de la valeur énergétique, en supposant que la chèvre ingère une ration mixte "moyenne " $(\mathrm{EM} / \mathrm{ED}=0,82, \mathrm{kl}=0,60)$. Ainsi, un besoin en énergie de $0,40 \mathrm{UFL} / \mathrm{kg}$ de lait à $35 \mathrm{p}$. 1000 de taux butyreux correspond à $342 \mathrm{~g}$ de MOD du foin moyen ingéré par les animaux, valeur supérieure de $6 \mathrm{p} .100 \mathrm{au}$ besoin habituel.

Les différences de niveau moyen d'ingestion à poids et production égaux entre les trois foins ne sont pas liées à leur digestibilité ou à leur composition. Elles semblent plutôt avoir pour origine le passé alimentaire et nutritionnel des animaux. Ce résultat soulève le problème de l'application du système des unités d'encombrement (UE) puisque les différences d'ingestion observées ne peuvent s'expliquer par les valeurs UE de ces foins.

Les valeurs de digestibilité sont identiques, à même composition d'ingéré, pour les animaux taris et ceux en lactation, quel que soit le niveau de production. La quantité de matière sèche ingérée n'a donc eu aucun effet sur l'utilisation digestive du foin comme Giger et al. (1986) l'ont observé sur des rations mixtes avec des chèvres ou FreDERIKSEN (1973) avec des vaches alimentées au prorata de leur niveau de production, mais contrairement aux données de Brown (1966) ou Tyrrell \& Moe (1975) où l'augmentation du niveau alimentaire entraînait une diminution de la digestibilité. Les différences de capacité d'ingestion déterminées par celles des besoins, pourraient être associées à des différences de développement du tube digestif permettant de ne pas modifier la durée de présence des particules dans les principaux réservoirs digestifs.

L'équation de prédiction de la digestibilité de la matière organique obtenue à partir de la relation intra-foin est précise puisque l'écart-type résiduel de la régression est de 1,6 point, ce qui est inférieur à 2 , valeur généralement admise comme valeur de l'incertitude expérimentale (Charlet-LeRY, 1969; RRI, 1975). Les « systèmes de Weende et de VAN SoEST » ont des pouvoirs de prédiction analogues de la digestibilité de la matière organique, ce qui peut s'expliquer par le fait que cette étude concerne une même espèce végétale (JARRIGE, 1981).

\section{Conclusion}

Avec un niveau moyen de refus de 15 p. 100, et pour un foin de luzerne de bonne qualité :

- Les chèvres taries ingèrent significativement moins (15 p. 100), mais digèrent mieux le foin de luzerne que les MCS, ce qui ne permet pas de leur appliquer les équations établies pour les moutons concernant l'ingestion et la digestibilité (INRA, 1981) ;

- Les chèvres, en triant le fourrage, améliorent sa valeur nutritive de 0,60 point de la dMO en moyenne, soit $0,01 \mathrm{UFL} / \mathrm{kg} \mathrm{MS}$; 
- Les variations du niveau d'ingestion du foin qui sont liées à celles du besoin de production n'ont pas d'influence sur le coefficient d'utilisation digestive des différentes fractions de ce foin, à composition d'ingéré égal ;

- Le foin de luzerne, d'une dMO voisine de 60 p. 100 , distribué seul assure une production laitière pouvant aller jusqu'à $2,8 \mathrm{~kg}$ de lait standard à $35 \mathrm{p}$. 1000 de taux butyreux, ce qui permet de subvenir aux besoins d'animaux en milieu et en fin de lactation.

Reçu en juin 1986.

Accepté en mars 1987.

\section{Summary \\ Nutritive value of lucerne hay for dairy goats}

Lucerne hay is an useful forage for dairy goats. Its nutritive value is well-known for sheep, but not for goats. It seemed therefore important to compare results obtained in these two species.

This study included seven different lucerne hay of medium to high nutritive value. They were given either to dry or lactating goats and their intake and digestibilty were measured (table 1). Dry goats ate significantly less $\left(-10.4 \mathrm{~g} / \mathrm{kg} \mathrm{BW}^{0.75}\right)$ than sheep (INRA, 1981) with the same level of refusal (about $15 \%$ ). Lactating goats ate more (about $33 \%$ ) than « standard sheep " (tabl. 2). Lactating goats digested the organic matter as well as dry goats, and both digested better (about 1.5 point of $\mathrm{OMD}$ ) than sheep.

The mean percentage of refusal was about $15 \%$ of the given feed with important individual variations (figure 1). The choice behaviour of goats improved the nutritive value of ingested hay with the reduction of its fibre content (figure 2 and tables 3 and 4) and the improvement of its crude protein content. The mean improvement of organic matter digestibility was 0.60 point. It was linked to the variations in fibre digestibility (table 5).

Milk production was not related to dry matter intake as far as all the data are concerned (figure 3). But within an experiment, milk production was highly correlated to hay intake. The observated differences between hays were not related to their digestibility, composition or to the energy requirements of animals, which gives rise to the problem of using ballast units.

Key words: Dairy goat, lucerne hay, nutritive value.

\section{Références bibliographiques}

Andrieu J., Demarquilly C., Wegat-Ltrre E., 1981. Tables de prévision de la valeur alimentaire des fourrages. In "Prévision de la valeur nutritive des aliments des ruminants" (ed. C. Demarquilly), 343-580, INRA publications, Route de Saint-Cyr, 78000 Versailles, (France).

Brown L.D., 1966. Influence of intake on feed utilization. J. Dairy Sci., 49, 223-230.

Brown L.E., Johnson W.L., 1984. Comparative intake and digestibility of forages and byproducts by goats and sheep : a review. Int. Goat Sheep Res., 2, 212-226.

Charlet-Lery G., 1969. Etude sur le questionnaire relatif aux méthodes de détermination des coefficients de digestibilité des aliments chez les ruminants. $20^{\mathrm{c}}$ Réunion annuelle de la FEZ, Helsinki, (Finlande), 1969/06/23-25, 33 p. 
De Simiane M., Giger S., Blanchart G., Huguet L., 1981. Valeur nutritionnelle et utilisation des fourrages cultivés intensément. In "Nutrition et systèmes d'alimentation de la chèvre " (ed. P. Morand-Fehr, A. Bourbouze, M. De Simiane), Symposium International sur les productions caprines, Tours, (France), 1981/05/12-15, 274-299, (vol. I), INRA-ITOVIC, Paris, (France).

Demarquilly C., Andrieu J., Sauvant D., 1978. Tableaux de la valeur nutritive des aliments. In "Alimentation des ruminants " (ed. R. JARRIGE), 519-555. INRA publications, Route de SaintCyr, 78000 Versailles, (France).

Frederiksen J.H., 1973. Method studies relating to digestibility trials with ruminants. Acta Agric. Scand., 23, 17-33.

Giger S., Hervieu J., 1980. Conception d'une cage à bilan adaptée à la chèvre en lactation. Ann. Zootech., 29, 55-64.

Giger S., Hervieu J., Dorleans M., Sauvant D., 1982. Protocole de travail pour les expériences de digestibilité effectuées au laboratoire de recherches (INRA) de la Chaire de Zootechnie de l'INA-PG. Document interne (5 p.).

Giger S., Sauvant D., Hervieu J., Dorleans M., 1986. Etude de la prévision de la digestibilité des rations mixtes distribuées à des chèvres laitières par ses caractéristiques analytiques. Ann. Zootech., 35, 137-160.

JARRIGE R., 1981. Les constituants glucidiques des fourrages : variations, digestibilité et dosage. In "Prévision de la valeur nutritive des aliments des ruminants" (ed. C. Demarquilly), 13-40, INRA publications, Route de Saint-Cyr, 78000 Versailles, (France).

Jones G.M., Larsen R.E., Jeveda H., Donefer E., Gaudreau J.M., 1972. Voluntary intake and nutrient digestibility of forages by goats and sheep. J. Anim. Sci., 34, 830-838.

Morand-Fenr P., 1981. Caractéristiques du comportement alimentaire et de la digestion des caprins. In "Nutrition et systèmes d'alimentation de la chèvre " (ed. P. Morand-FenR, A. Boubouze, M. De Simiane), Symposium International sur les productions caprines, Tours, (France), 1981/05/12-15, 21-45, (vol. I), INRA-ITOVIC, Paris, (France), 544 p.

Morand-Fehr P., Giger S., Sauvant D., Broqua B., De Simiane M., 1987. Utilisation des fourrages secs par les caprins. In * Les fourrages secs : récolte, traitement, utilisation „ (ed. C. Demarquilly). XVI ${ }^{\text {ss }}$ Journées du Grenier de Theix, Clermont-Ferrand, (France), 391-422, INRA publications, Paris, (France), $689 \mathrm{p}$.

RRI, Rowett Research Institute, 1975. Feedingstuffs evaluation unit. First report 1975, DAFS, Edimburgh, (Royaume-Uni), $58 \mathrm{p}$.

SaUvant D., 1981. Alimentation énergétique des caprins. In "Nutrition et systèmes d'alimenation de la chèvre " (ed. P. Morand-Fehr, A. Bourbouze, M. De Simiane), Symposium International sur les productions caprines, Tours, (France), 1981/05/12-15, 55-79, (vol. I), INRAITOVIC, Paris, (France), 544 p.

Sauvant D., Giger S., Chiluiard Y., Morand-Fehr P., 1981. Etude de la capacité d'ingestion de la chèvre en début de lactation. In "Nutrition et systèmes d'alimentation de la chèvre " (ed. P. Morand-Fehr, A. Bourbouze, M. De Simiane), Symposium International sur les productions caprines, Tours, (France), 1981/05/12-15, 572-581, (vol. II), INRA-ITOVIC, Paris, (France), 764 p.

Snedecor G.W., Cochran W.G., 1984. Méthodes statistiques, Sixième édition, Acta, Paris, (France), 649 p.

Tyrrell H.F., MoE P.W., 1975. Effect of intake on digestive efficiency. J. Dairy Sci., 58, 11511163.

VAN SoEst P.J., 1963. Use of detergents in the analysis of fibrous feeds. II/ A rapid method for the determination of fibre and lignin. J. Assoc. off. anal. Chem., 46, 829-835.

VAN Soest P.J., WINE R.H., 1967. Use of detergents in the analysis of fibrous feeds. V/ Determination of plant cell-wall constituents. J. Assoc. off. Anal. Chem., 50, 50-55. 